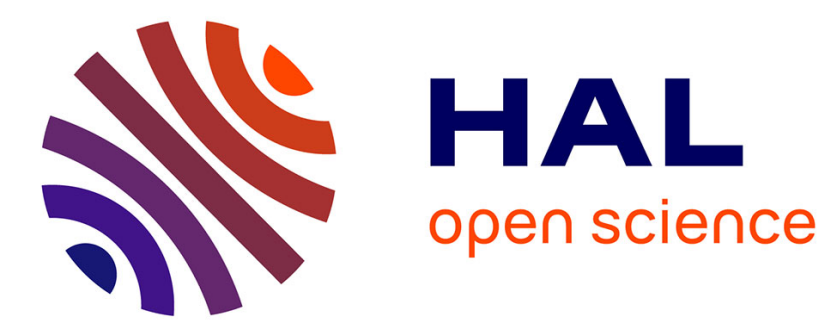

\title{
Plasma oscillations in heavy-fermion materials
}

A. J. Millis, M. Lavagna, P.A. Lee

\section{To cite this version:}

A. J. Millis, M. Lavagna, P.A. Lee. Plasma oscillations in heavy-fermion materials. Physical Review B: Condensed Matter (1978-1997), 1987, 36 (1), pp.864-867. 10.1103/PhysRevB.36.864 . hal-01896336

\section{HAL Id: hal-01896336 https://hal.science/hal-01896336}

Submitted on 16 Oct 2018

HAL is a multi-disciplinary open access archive for the deposit and dissemination of scientific research documents, whether they are published or not. The documents may come from teaching and research institutions in France or abroad, or from public or private research centers.
L'archive ouverte pluridisciplinaire HAL, est destinée au dépôt et à la diffusion de documents scientifiques de niveau recherche, publiés ou non, émanant des établissements d'enseignement et de recherche français ou étrangers, des laboratoires publics ou privés. 


\title{
Plasma oscillations in heavy-fermion materials
}

\author{
A. J. Millis \\ AT\&T Bell Laboratories, 600 Mountain Avenue, Murray Hill, New Jersey 07974 \\ M. Lavagna \\ Laboratoire Louis Néel, Centre National de la Recherche Scientifique, Boîte Postale No. 166x, \\ 38042 Grenoble Cedex France \\ P. A. Lee \\ Department of Physics, Massachusetts Institute of Technology, Cambridge, Massachusetts 02139
}

(Received 2 February 1987)

\begin{abstract}
We calculate the dielectric function of the lattice Anderson model via an auxiliary-boson large- $N$ method suitably generalized to include the effects of the long-range part of the Coulomb interaction. We show that the model exhibits a low-lying plasma oscillation at a frequency $\omega^{*}$ on the order of the Kondo temperature of the model, in addition to the usual high-frequency plasma oscillation. We also analyze the Anderson model without the long-range Coulomb interaction, computing the Landau parameter $F_{0 s}$, and showing that the model has a zero-sound mode whose velocity we compute. We derive the version of the $f$ sum rule applicable to our model and show that our results satisfy it.
\end{abstract}

Heavy-electron metals are a class of compounds involving rare-earth or actinide elements which have recently been much studied ${ }^{1,2}$ because at low temperatures their properties are, crudely speaking, those of a Fermi liquid of mass $m^{*} \sim 10^{2} m$ ( $m$ is the usual electron mass) and Fermi temperature $T^{*} \sim 10-100 \mathrm{~K}$. Here we report results of a theoretical study of the small- $q$ limit of the dielectric function $\epsilon(q, \omega)$. Our principle result is that heavyelectron metals should have a plasmon mode at the renormalized plasma frequency $\omega^{*} \cong T^{*}$. The plasmon has low spectral weight, making an order $\mathrm{m} / \mathrm{m}^{*}$ contribution to the $f$ sum rule, and is likely to be damped, but perhaps ought to be observable in reflectivity or other measurements. We also relate our results to a previous calculation $^{3}$ of the optical conductivity $\sigma(\omega)$, and comment on the implication of our results for the physical interpretation of the heavy-fermion compounds.

As a model for the low-temperature behavior of heavyelectron materials we use the auxiliary-boson (or "slaveboson") large- $N$ version of the lattice Anderson model. The Anderson model is believed ${ }^{2}$ to represent the essential physics of heavy-fermion materials. The slave-boson, large- $N$ version was devised for the single-impurity Anderson model ${ }^{4-6}$ and has been also used to study various aspects of the lattice problem. ${ }^{3,7-10}$ For a comparison of this with other methods, see Ref. 2.

The Anderson model describes a structureless band of conduction electrons (operator $c_{k \sigma}$, energy $\varepsilon_{k}$ ) hybridizing via a hybridization matrix element $V$ (conventionally assumed to be structureless), with a dispersionless band of $f$ electrons at an energy $E_{0}$, and subject to the constraint that the number of $f$ electrons on site $i, n_{f i} \leq 1$. We measure all energies with respect to the chemical potential (taken to be zero) and we are interested in the Kondo limit in which $-E_{0} / \rho_{0} V^{2} \gg 1 . \rho_{0}$ is the $c$-electron density of states evaluated at $\varepsilon_{k}=0$. We assume $T=0$ throughout.
The Anderson model as conventionally formulated does not include the (physically necessary) long-range part of the Coulomb interaction. We therefore add to it a term of the form

$$
H_{\mathrm{Coul}} \sim \sum_{q<q_{c}} \frac{4 \pi e^{2}}{q^{2}} n_{q} n_{-q},
$$

where $n_{q}$ is the Fourier transform of the density $n_{i}=n_{c i}+n_{f i}$. We note that the short-range part of the Coulomb interaction between the $f$ electrons is already included in the Anderson model, where it produces the "infinite- $U$ " repulsion leading to the constraint $n_{f i} \leq 1$. However, the long-range part of this interaction is not included, as can be seen from a Gedankenexperiment in which a few $f$ electrons are moved from one end of the lattice to the other, with everything else held fixed. The sum on $q$ in $H_{\text {Coul }}$ must be cut off at $q=q_{c} \ll k_{F}$ to avoid double counting. In this Rapid Communication we are concerned only with asymptotically-long-wavelength properties, and will never have to specify $q_{c}$.

The inequality constraint on $n_{f}$ makes the model difficult to attack by conventional methods. In the slaveboson method one introduces a new boson field $b_{i}$ representing an empty set of $f$ orbitals on site $i$, rewrites the hybridization $\left(V c_{i}^{\dagger} f_{i} b_{i}^{\dagger}+\right.$ H.c. $)$ and enforces the constraint $n_{f i}+n_{b i}=1$ via a Lagrange multiplier field $\lambda$. To use the $1 / N$ expansion one assumes both $c$ and $f$ electrons are characterized by a $N$-fold-degenerate "spin" quantum number $m$, conserved in hybridization and $c$-electron propagation, and one rewrites the constraint as $n_{f i}$ $+n_{b i}=q_{0} N$, where $q_{0}$ is regarded formally as independent of $N$. (To recover the original Anderson model one sets $q_{0} \rightarrow 1 / N$ at the end of any calculation. ${ }^{3}$ ) Next, one splits the boson operators into static and fluctuating parts. $\mathrm{Re}-$ taining only the static parts leads to a mean-field theory of electrons moving in a renormalized band structure in 
which a dispersionless band of $f$ quasiparticles ${ }^{3}$ at energy $T^{*}>0$ hybridizes via a renormalized hybridization $\sigma_{0} \ll V$ with the $c$-electron band. Corrections to the mean-field theory come from Coulomb interactions and interactions between the electrons and the fluctuating parts of the boson fields. To study these corrections we use the radial gauge formulation, ${ }^{5}$ but we write the electrons in terms of the operators $d_{i}$ which diagonalize the mean-field theory, and we take the small- $q$ limit in the interaction terms. The model is then specified by the Lagrangian $L=L_{F}+L_{B}+L_{I}+L_{\text {Coul }}+L_{\text {rest }}$, where

$$
\begin{aligned}
L_{F}= & \sum_{i, k, m} d_{i k m}^{\dagger}\left[\partial_{\tau}+\varepsilon_{i}(k)\right] d_{i k m}, \\
L_{B}= & \frac{N}{2 V^{2}} \sum_{q} \sigma_{q}\left(T^{*}-E_{0}\right) \sigma_{q}+2 i \sigma_{0} \sigma_{q} \lambda-q, \\
L_{I}= & \sum_{k, q, m} d_{1, k+q, m}^{\dagger} d_{1 k m}\left(\frac{2 \sigma_{0}}{E_{k}} \sigma_{q}+i \lambda_{q}\right) \\
& +\left(d_{2 k m}^{\dagger} d_{1, k+q, m}+\text { H.c. }\right)\left(\frac{\varepsilon_{k}-\varepsilon_{f}}{E_{k}} \sigma_{q}-\frac{i \sigma_{0}}{E_{k}} \lambda_{q}\right),
\end{aligned}
$$

where the $d_{1(2) k m}$ and $\varepsilon_{1(2) k}(k)$ are the operators and energies for the lower and upper bands of the renormalized band structure; one has

$$
\varepsilon_{1(2)}(k)=\frac{1}{2}\left[\left(\varepsilon_{k}+T^{*}\right)-(+) E_{k}\right]
$$

and

$$
E_{k}=\sqrt{\left(\varepsilon_{k}-T^{*}\right)^{2}+4 \sigma_{0}^{2}} .
$$

We have assumed the Fermi level lies in the lower band. The $d_{k i}$ are related to the $c$ and $f$ operators via

$$
d_{k i}=\cos \theta_{k i} c_{k i}+\sin \theta_{k i} f_{k i},
$$

where

$$
\tan \theta_{k i}=\left[\varepsilon_{i}(k)-\varepsilon_{k}\right] / \sigma_{0}=\sigma_{0} /\left[\varepsilon_{i}(k)-T^{*}\right] .
$$

The mean-field parameters $\sigma_{0}$ and $T^{*}$, to leading order in $1 / N$, and $q_{0}$ are given by ${ }^{3} T^{*}=D \exp \left(E_{0} / \rho_{0} V^{2}\right)$, $\sigma_{0}^{2}=q_{0} V^{2}\left(1-n_{f}\right)$, and $n_{f}=\left(1+T^{*} / \rho_{0} V^{2}\right)^{-1}$. Here $D$ is an energy of the order of the distance of the bottom of the $c$-electron band from the chemical potential. The density of states at the Fermi surface $\varepsilon_{1}(k)=0$ is $\left(m^{*} / m\right)$ $\times\left(k_{F}^{2} / 2 \pi^{2}\right)\left(d k / d \varepsilon_{k}\right)$, where $m^{*} / m=\sigma_{0}^{2} /\left(T^{*}\right)^{2}=\varepsilon_{k_{F}}^{2} / \sigma_{0}^{2}$. The band structure defined by $L_{F}$ is thus very flat at $k_{F}$; its excitations are heavy fermions of velocity $v^{*}=d \varepsilon_{1}\left(k_{F}\right) / d k$. The band structure has a direct gap of magnitude $2 \sigma_{0}$ centered at $\varepsilon_{k}=T^{*}$.

The operators $\sigma_{q}$ and $\lambda_{q}$ are related to the fluctuating part of the original Bose operators. In terms of the operators $d_{i}$, the density operator is

$$
n_{q}=\sum_{k, m} d_{1, k+q, m}^{\dagger} d_{1 k m}+\mathbf{p}_{k} \cdot \mathbf{q}\left(d_{2, k+q, m}^{\dagger} d_{1 k m}+\text { H.c. }\right)
$$

$\mathbf{p}_{k}=\left(\sigma_{0} / E_{k}^{2}\right)\left(d \varepsilon_{k} / d \mathbf{k}\right)$ is the dipole operator which gives rise to interband transitions.

The factor of $N$ in $L_{\text {Coul }}$ arises because we consider a model with $\sim 1$ electron per spin channel.

The expressions above are correct only up to terms of relative order $\left(q / k_{F}\right)^{2}$. The terms in $L_{\text {rest }}$, which we have not explicitly written, include a three-boson interaction (which does not contribute to the order to which we work) with the terms which fix the mean-field parameters, and the terms involving band-2 operators which do not contribute at $T=0$.

From Eqs. (1)-(4) one may easily compute the density-density correlation function $\chi(q, \omega)$ by standard diagrammatic techniques. Details of a similar calculation are given in Ref. 10. To leading order in $1 / N$ the only diagrams which contribute are shown in Fig. 1. They have the familiar random-phase-approximation (RPA) form of polarization bubbles connected by interaction lines, which in this case may be either boson propagators [from Eq. (1b)] or the Coulomb interaction. The polarization bubbles may be of inter- or intra-band type. Note that the density operator (and thus the Coulomb interaction) couples to an interband bubble via a vector vertex, but the boson propagator couples to an interband bubble via a scalar vertex. Summing the diagrams we find at small $q$,

$$
\chi(q, \omega)=-\frac{\Pi(q, \omega)+\Pi_{12}^{0}(q, \omega)}{1+\left(4 \pi e^{2} / q^{2} N\right)\left[\Pi(q, \omega)+\Pi_{12}^{0}(q, \omega)\right]} .
$$

Here $\Pi_{12}^{0}$ is the interband density polarization bubble,

$$
\begin{aligned}
\Pi_{12}^{0}(q, \omega)=-N \sum_{k\left(<k_{F}\right)}\left(\mathbf{p}_{k} \cdot \mathbf{q}\right)^{2} \\
\times\left(\frac{1}{\omega-E_{k}+i \delta}+\frac{1}{-\omega-E_{k}-i \delta}\right),
\end{aligned}
$$

and $\Pi(q, \omega)$ is the intraband density polarization bubble, which includes the effects of electron-slave-boson interactions. One finds

$$
\Pi(q, \omega)=\Pi_{0}(q, \omega) / 1+\Gamma_{B}(\omega) \Pi_{0}(q, \omega),
$$

where $\Pi_{0}$ is the bare intraband polarization bubble;

$$
\Pi_{0}(q, \omega)=-N \sum_{k} \frac{f\left(\varepsilon_{1}(k)\right)-f\left(\varepsilon_{1}(k+q)\right)}{\omega+\varepsilon_{1}(k)-\varepsilon_{1}(k+q)+i \delta} .
$$

$\Gamma_{B}$ is the effective boson-mediated interaction between two band-1 quasiparticles with $k \cong k_{F}$. It is made up of

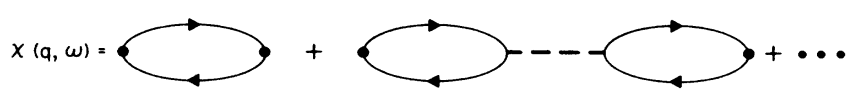

FIG. 1. Diagrams for density-density correlation function, to leading order in $1 / N$. The heavy dots denote density vertices. The solid lines with arrows represent electron Green's functions obtained from the inverse of $L_{F}$, Eq. (2a) in the text. The dashed line represents either bare boson propagators obtained from inverting $L_{B}$, Eq. (2b) in the text, or the Coulomb interaction $4 \pi e^{2} / q^{2} N$. The vertices at which boson or Coulomb lines join fermion lines are obtained from $L_{l}$, Eq. (2c) and $L_{c}$, Eq. (2d), respectively. 
bare boson propagators appropriately dressed by interband bubbles. $\Gamma_{B}$ is a function of $\omega / \sigma_{0}$ and we shall require only its $\omega \rightarrow 0$ limit, which we find to be $\Gamma_{B}(0)=\left(m^{*} / m \rho\right)$.

Equation (3) is the central result of this paper. To understand it, we first study the model without long-range electric fields by setting $e^{2}=0$. Equation (3) then becomes $\chi=\Pi+\Pi_{12}^{0}$. The term in $\Pi_{12}^{0}$, which is explicitly of order $q^{2}$, gives the contribution to $\chi$ from interband transitions. The term in $\Pi$ gives the intraband contribution, which is altered from the "noninteracting" value $\Pi_{0}$ by the electron-boson interactions. By using Eqs. (3)-(6) we find $\lim _{q \rightarrow 0} \chi(q, 0)=\rho\left(1 / 1+\rho \Gamma_{B}\right)$. We therefore identify $\rho \Gamma_{B}(\omega=0)$ with the Landau parameter $F_{0 s}=m^{*} / m \gg 1$. All other Landau $F$ parameters are of order $1 / N .^{3,8}$ Note also that $\chi$ has a pole at $\omega^{2}=c^{2} q^{2}$, with $c^{2}=\left(F_{0 s} / 3\right) v^{* 2}$; this is the familiar zero-sound mode of a neutral Fermi liquid with $F_{0 s} \gg 1$. The Fermi liquid under consideration stems from the hybridization of two bands, one of which is dispersionless. The model is therefore not Galilean invariant. In a Galilean-invariant Fermi liquid with $m^{*} / m \gg 1$, the expression for the zero-sound velocity would necessarily involve also $F_{1 s}=3\left[\left(m^{*} / m\right)-1\right]$.

Consider now the spectral weight of density-fluctuation excitations, $S(q, \omega)=(1 / \pi) \operatorname{Im} \chi(q, \omega) . \quad S(q, \omega)$ satisfies the $f$ sum rule ${ }^{11}$

$$
\int_{0}^{\infty} d \omega \omega S(q, \omega)=n_{\text {tot }} q^{2} / 2 m
$$

where $n_{\text {tot }}$ is the total number of electrons and $m$ is the electron mass. The Anderson model, however, is an effective Hamiltonian presumed to describe the physics of heavy-fermion materials at energies less than the conduction-electron bandwidth, which is of order $D$. In particular, higher-excited states of the $f$ electrons, which presumably form bands at energies $\gtrsim D$ above the Fermi surface, are not included. These higher bands will contribute, at high frequencies, to the $f$ sum rule; therefore, the $S$ derived from Eq. (3) does not satisfy the full $f$ sum rule. However, by applying the standard derivation ${ }^{11}$ of the $f$ sum rule to the Anderson model we have derived a partial $f$ sum rule

$$
\int_{0}^{\infty} d \omega \omega S(q, \omega)=n_{c} q^{2} / 2 m
$$

Here $n_{c}$ is the number of conduction electrons. (We assume unit volume.) If one assumes $\varepsilon_{k}=k^{2} / 2 m-\mu$, then $n_{c}=N k_{h}^{3} / 6 \pi^{2}$ where $k_{h}=\sqrt{2 m \mu}$. We expect that the difference $\left(n-n_{c}\right) q^{2} / 2 m$ between (7) and the full $f$ sum rule is made up by the previously mentioned interband contributions to $S(q, \omega)$ at $\omega \gtrsim D$.

Now using Eq. (3) one may easily check that (7) is satisfied at small $q$. In the case $e^{2}=0$ there are three contributions. One comes from the particle-hole continuum near the Fermi surface, $\omega<v^{*} q$, and contributes a term of order $n_{c} q^{2} / F_{0 s}^{2} m^{*}$ to the right-hand side of (7). Thus the low-lying quasiparticle density fluctuations have negligible spectral weights. The second contribution comes from the zero-sound mode; it contributes $n q^{2} / 2 m^{*}$, where in a model with a spherical Fermi surface, $n=N k_{F}^{3} / 6 \pi^{2}$. The third contribution comes from the interband transitions at frequencies $\omega \gtrsim 2 \sigma_{0}$ contained in $\Pi_{12}^{0}$, yielding $n_{c} q^{2} / 2 m-\left(n q^{2} / 2 m^{*}\right)$.
It is now accepted that the low-energy excitations about the $T=0$ ground state of the lattice Anderson model are those of a Fermi liquid of large effective mass $m^{*} .2,3,8,12$ It has also been asserted that the effective density of particles in the Fermi liquid is $n\left(\mathrm{~m} / \mathrm{m}^{*}\right){ }^{12}$ One may interpret the results of the present paper as supporting this assertion, because the "Fermi-liquid" contributions (from the intraband particle-hole continuum and the zero-sound mode) to $S(q, \omega)$ contribute only $n\left(m / m^{*}\right) q^{2} / 2 m$ to the total spectral weight required by the $f$ sum rule. However, it is also argued in Ref. 12 that $n\left(m / m^{*}\right) \sim\left(1-n_{f}\right)$, so that heavy fermions are to be interpreted as holes in the $f$ band. This assertion is not supported by the present model because $n_{f}$ and $m^{*} / m$ are separate parameters of the model and can be independently varied by appropriate variations of the bare parameters $E_{0}$ and $V$.

We now consider the physically relevant model, in which long-range Coulomb forces are present. For charged systems the relevant quantity is the dielectric function, $\epsilon(q, \omega)$, which may be computed either directly from the diagrams for the dressed Coulomb interaction or from the identity

$$
\epsilon^{-1}(q, \omega)=1+\left(4 \pi e^{2} / q^{2}\right) \chi(q, \omega) .
$$

By either method one finds

$$
\epsilon(q, \omega)=1+\frac{4 \pi e^{2}}{q^{2}}\left[\Pi(q, \omega)+\Pi_{12}^{0}(q, \omega)\right] .
$$

In the static limit, $\omega=0, \Pi(q, \omega) \rightarrow \rho / 1+F_{0 s}$, while $\Pi_{12}^{0}$ tends to the value $\left(q^{2} / 4 \pi e^{2}\right)\left(\omega_{p c}^{2} / 6 \sigma_{0}^{2}\right)$, where $\omega_{p c}^{2}=4 \pi n_{c} e^{2 / m}$. Thus

$$
\epsilon(q, 0)=\epsilon_{0}+\frac{4 \pi e^{2}}{q^{2}}\left(\frac{\rho}{1+F_{0 s}}\right) .
$$

Here $\epsilon_{0}=1+\omega_{p c}^{2} / 6 \sigma_{0}^{2}$. In physical terms $\epsilon_{0}$ represents a reduction in the Coulomb interaction between band-1 quasiparticles due to polarization of the "light" $c$-electron degrees of freedom. Because $F_{0 s}=m^{*} / m$, the ThomasFermi screening length is not renormalized from a value characteristic of a conventional light-electron system.

The frequency-dependent conductivity $\sigma(\omega)$ is related to the dielectric function by

$$
\lim _{q \rightarrow 0} \epsilon(q, \omega)=1+4 \pi i \sigma(\omega) / \omega .
$$

The results presented here for $\epsilon(q, \omega)$ agree with results previously calculated ${ }^{3}$ for $\sigma(\omega)$ in the limit $N \rightarrow \infty$, $\tau \rightarrow \infty$ ( $\tau$ is the impurity scattering time defined in Ref. 3).

Zeros of $\epsilon(q, \omega)$ correspond to plasma oscillations of the system. There are two in this model, at $\omega=\omega_{\text {high }}$ and $\omega=\omega^{*}$, where, using Eqs. (3)-(5) and (8),

$$
\begin{aligned}
& \omega_{\text {high }}^{2}=\omega_{p c}^{2}-O\left(m / m^{*}\right), \\
& \omega^{* 2}=6\left(1+n_{f} / n_{c}\right) T^{* 2} .
\end{aligned}
$$

The high-frequency plasma oscillation occurs at approximately the plasma frequency of the $c$ electrons alone. This is to be expected: Heavy-fermion behavior is essentially a low-frequency and -temperature phenomenon, which should not affect high-frequency phenomena such 
as the plasma oscillation.

The low-frequency oscillation may be thought of as a heavy fermion plasma mode. It is reduced from a typical plasma frequency $\omega_{p}^{2}=4 \pi e^{2} n / m$ by two effects: (i) the mass enhancement $\left(m^{*} / m\right)$ and (ii) the reduction of the effective Coulomb interaction between two band-1 quasiparticles which is given by $\epsilon_{0}$. The low-frequency plasma oscillation may be thought of as the zero-sound oscillation of the neutral system, pushed up to a finite frequency by the long range of the Coulomb interaction. It has spectral weight $n q^{2} / 2 m^{*}$ as does the zero-sound mode.

The form for $\epsilon$ is precisely what would be expected for a metal which happened to have the "mean-field" band structure described by Eq. (1a). ${ }^{13}$ However, the meanfield solution is valid only for $T \ll T^{*}$; for larger $T$ a picture in which $c$ electrons are incoherently scattered by spin fluctuations is more appropriate. Our theory applies only for very low temperatures, but we suspect that $\operatorname{Re} \epsilon$ would not have a low-lying zero crossing for temperatures comparable to or greater than $T^{*}$.

Zeroes of $\epsilon$ correspond to poles in $\chi$. By using Eq. (3) with for the charged case one easily verifies that to leading order in $q^{2}$ the only contributions to (7) come from the two plasmon poles. The plasmon at $\omega^{*}$ contributes $n q^{2} / 2 m^{*}$; the plasmon at $\omega_{\text {high }}$ contributes $n_{c} q^{2} / 2 m$ $-n q^{2} / 2 m^{*}$. Thus the low-lying plasmon has spectral weight $n q^{2} / 2 m^{*}$ as did the zero sound mode of the neutral system. Note that $\operatorname{Re} \epsilon(q, \omega)$ also vanishes at a value $\omega=\omega_{0} \gtrsim 2 \sigma_{0}$. However, at $\omega=\omega_{0}$, Im $\epsilon$ is large because of interband transitions. This zero of $\operatorname{Re} \epsilon$ therefore corresponds to a heavily damped oscillation, and not to a distinguishable mode of the system. The other two plasma modes are undamped, to leading order in $1 / N$.
To summarize, we have calculated the dielectric function for the Anderson lattice to leading order in $1 / N$ using the slave-boson method. We have included the long-range part of the Coulomb interaction. We have shown that the model exhibits a low-frequency plasma oscillation at a frequency $\omega^{*} \sim T^{*}$, where $T^{*}$ is the characteristic or Kondo temperature of the model, as well as the usual high frequency plasmon.

In conclusion, we discuss the observability of the lowlying plasmon in real heavy-fermion materials. We first note that the characteristic energy scale is set by $T^{*}$, the Fermi temperature of the heavy fermions. $T^{*}$ determines the coefficient of the linear term in the low-temperature specific heat and the $T^{2}$ term in the resistivity. ${ }^{3} T^{*}$ determines the heavy-fermion plasma frequency $\left(n_{f} / n_{c}\right.$ is of the order 1), and our calculation only applies for temperatures small compared to $T^{*}$. In the very-low-temperature regime where our calculation is valid, the low-lying plasmon is undamped to leading order in $1 / N$. However, the system is not Galilean-invariant; therefore $1 / N$ effects involving inelastic scattering of electrons off of slaveboson fluctuations could, in a system in which $N$ is not large, lead to a large value of $\operatorname{Im} \epsilon(q, \omega)$ for $\omega \sim T^{*}$. This would substantially broaden the low-lying plasmon pole (and, also, the interband edge). The broadening, combined with the low spectral weight, may make the plasmon difficult to observe.

This work is a result of a collaboration begun when the authors were at MIT, whose Physics Department we thank for hospitality. One of us (P.A.L.) acknowledges National Science Foundation Grant No. NSF-DMR8521377 for support.
${ }^{1}$ For a review of experimental work up to 1984 , see G. R. Stewart, Rev. Mod. Phys. 56, 755 (1984).

${ }^{2}$ P. A. Lee, T. M. Rice, J. W. Serene, L. J. Sham, and J. W. Wilkins, Comments Condens. Matter Phys. 12, 99 (1986).

${ }^{3}$ A. J. Millis and P. A. Lee, Phys. Rev. B 35, 3394 (1987).

${ }^{4}$ S. E. Barnes, J. Phys. F 6, 1375 (1976).

${ }^{5}$ P. Coleman, Phys. Rev. B 29, 3035 (1984).

${ }^{6}$ N. Read and D. M. Newns, J. Phys C 16, 3273 (1983).

${ }^{7}$ N. Read, D. M. Newns, and S. Doniach, Phys. Rev. B 30, 3841 (1984).
${ }^{8}$ A. Auerbach and K. Levin, Phys. Rev. Lett. 57, 877 (1986).

${ }^{9}$ M. Lavagna, A. J. Millis, and P. A. Lee, Phys. Rev. Lett. 58, 266 (1987).

${ }^{10}$ A. J. Millis, Phys. Rev. B (to be published).

${ }^{11}$ D. Pines and P. Nozieres, Theory of Quantum Liquids (Benjamin, New York, 1966), pp. 90ff and $219 \mathrm{ff}$.

${ }^{12}$ T. M. Rice and K. Ueda, Phys. Rev. B 34, 6420 (1986).

${ }^{13}$ D. Pines and P. Nozieres, Ref. 11, p. $221 \mathrm{ff}$. 